\title{
BMJ Open Effects of socioeconomic status on the control of hypertension in patients $<65$ and $\geq 65$ years of age in Taiwan: a nationwide cross-sectional study
}

\author{
Sheng-Feng Lin, ${ }^{1,2,3,4}$ Tzu-Tung Kuo, ${ }^{2}$ WH Pan, ${ }^{5}$ Chyi Huey Bai (1) 1,2,6
}

To cite: Lin S-F, Kuo T-T, Pan WH, et al. Effects of socioeconomic status on the control of hypertension in patients $<65$ and $\geq 65$ years of age in Taiwan: a nationwide cross-sectional study. BMJ Open 2022;12:e050041. doi:10.1136/ bmjopen-2021-050041

- Prepublication history and additional supplemental material for this paper are available online. To view these files, please visit the journal online (http://dx.doi.org/10.1136/ bmjopen-2021-050041).

Received 10 February 2021 Accepted 18 December 2021

Check for updates

(C) Author(s) (or their employer(s)) 2022. Re-use permitted under CC BY-NC. No commercial re-use. See rights and permissions. Published by BMJ.

For numbered affiliations see end of article.

Correspondence to

Dr Chyi Huey Bai;

baich@tmu.edu.tw

\section{ABSTRACT}

Objective Implementation of National Health Insurance in 1995 improved the control of hypertension due to comprehensive coverage of medical costs for all residents in Taiwan. However, the role of individual socioeconomic status (iSES), namely, education and personal income, in awareness, treatmen, and control of blood pressure, has not been well studied in Taiwan.

Design A nationwide cross-sectional survey was executed in this study.

Setting A systematic, multistage sampling scheme from a nationwide cohort in Taiwan was adopted to select adult participants. Hypertension was defined as blood pressure $\geq 140 / 90 \mathrm{~mm} \mathrm{Hg}$ or the use of antihypertensive medications.

Participants A total of 4599 subjects completed door-todoor household interviews and physical examinations.

Primary and secondary outcome measures A summing $z$ score of iSES was constructed by two domains of the iSES, namely, income and education, through which participants were classified into three SES levels. Logistic regression models were used to assess associations of awareness, treatment, and control in younger ( $<65$ years) and older ( $\geq 65$ years) participants with hypertension.

Results Younger people in the highest tertile of iSES were less likely to be aware of their hypertension (OR: 0.48, $95 \% \mathrm{Cl}: 0.37$ to $0.64, \mathrm{p}<0.0001)$ or to have it treated (OR: $0.49,95 \% \mathrm{Cl} 0.37$ to $0.64, \mathrm{p}<0.0001$ ) but more likely to have their hypertension controlled (OR: $1.52,95 \% \mathrm{Cl} 1.19$ to $1.94, p=0.0009$ ). We did not detect an association in people aged $>65$ years.

Conclusion Health education to improve awareness and treatment of hypertension should focus on younger people with higher iSES in Taiwan.

\section{INTRODUCTION}

The initial nationwide surveillance data for the control of blood pressure (BP) in Taiwan were derived from the Nutrition and Health Survey in Taiwan (NAHSIT) 1993-1996, ${ }^{1}$ and the second set of surveillance data was analysed from a database of the Taiwanese Survey on Hypertension, Hyperglycaemic and Hyperlipidemia study in 2002. ${ }^{2}$ Between the period of conduct of the two surveys, significant

\section{Strengths and limitations of this study}

- The nationwide study included a large number of representative Taiwanese participants with adequate statistical power.

- Two dimensions of socioeconomic status (SES), namely, income and education, were considered simultaneously with a summing of $z$ score.

- Sensitivity analysis confirmed the robustness of the study after considering factors such as diabetes, chronic kidney disease, urbanisation and smoking status.

- A direct causal relationship between hypertension control and SES was observed due to the crosssectional design.

- Knowledge of hypertension was not assessed in the survey.

improvements in the control of hypertension were found due to the implementation of National Health Insurance (NHI) in $1995,{ }^{2}$ which covers almost all $(>99.9 \%)$ of the residents of Taiwan, including all medical costs of in-hospital and out-of-hospital treatment of hypertension and hypertension-related diseases. The NHI in Taiwan has been established for 25 years and is ongoing.

Based on epidemiological studies and clinical evaluations, it is known that hypertension is strongly associated with stroke and ischaemic heart diseases. ${ }^{3}{ }^{4}$ Globally, hypertension and cardiovascular diseases cause one-third of deaths. ${ }^{5}$ In Taiwan, hypertension, ischaemic heart disease and stroke have long remained three of the top 10 leading causes of death. ${ }^{6}$ In general, a lower socioeconomic status (SES) is considered to be associated with higher $\mathrm{BP}^{7-9}$ and poor awareness of hypertension due to limited medical availability and resources. Since the NHI in Taiwan offers readily available and equal medical resources throughout all the SES classes and residential regions, we considered 
that the role of SES should be studied accordingly. Thus, we attempted to investigate whether SES at the individual level (iSES) still plays a pivotal role in awareness, treatment and control of hypertension by accessing data from the NAHSIT 2013-2016 survey.

\section{METHODS}

\section{Patient and public involvement}

In this nationwide survey of behavioural indicators and health outcomes, we investigated participants' health status in Taiwan. The NAHSIT 2013-2016 is a populationbased cross-sectional survey of the health and nutritional status of the residents of Taiwan. ${ }^{10}{ }^{11}$ Participants were systemically sampled from among 359 districts and townships nationwide in Taiwan according to population density, geographical area and degree of urbanisation. ${ }^{10}{ }^{11}$ In addition, strata within districts and townships were selected by a method of probability proportional to their size. A door-to-door interview survey and physical examination of each household were conducted by trained interviewers. After obtaining consent for phlebotomy, serum samples for serum creatinine, fasting glucose and glycated haemoglobin Alc (HbAlc) were examined in a central laboratory. According to the standards of the American Diabetes Association, ${ }^{12}$ participants were classified as having diabetes mellitus (DM) when they had fasting glucose $\geq 126 \mathrm{mg} / \mathrm{dL}$ or HbA1c level $\geq 6.5 \%$. Kidney function as assessed by the estimated glomerular filtration rate (eGFR) was determined by serum creatinine using the Chronic Kidney Disease Epidemiology Collaboration (CKD-EPI) formula. ${ }^{13}$ Since stage 3 or more advanced CKD could be identified by serum creatinine alone, ${ }^{14-16}$ participants were classified as having chronic kidney disease (CKD) by eGFR of $<60$ $\mathrm{mL} / \mathrm{min} / 1.73 \mathrm{~m}^{2}$. Smoking status was assessed by two questions in the Chinese language: (1) 'have you ever smoked in your lifetime?' and (2) 'do you smoke now?' Accordingly, participants were categorised into current, former and never smokers.

\section{Definition of older adults in Taiwan}

The WHO defines a society in which the proportion of people aged $\geq 65$ years exceeds $7 \%$ as an 'ageing society'. When this proportion surpasses $14 \%$ in a society, it is regarded as an 'aged society'. Accordingly, the prevalence of older adults $\geq 65$ in Taiwan exceeded $14 \%$ in $2018 .{ }^{17}$ In the last decade, policies on health education by the Ministry of Health and Welfare in Taiwan ${ }^{18}$ have focused on older adults aged $\geq 65$ years.

\section{Measures of BP}

All participants were asked to remain relaxed and to refrain from exercise, smoking and drinking coffee or tea within 30 min before BP measurement. The brachial BPs were measured with an appropriately sized cuff at the heart level, and participants were asked to be seated in a quiet room and relax for $5 \mathrm{~min}$ in a position with the arms supported and the legs uncrossed. BP measurements were obtained by certified interviewers, and if two of the three $\mathrm{BP}$ measurements varied by $\geq 10 \mathrm{~mm} \mathrm{Hg}$, a fourth measurement was made accordingly. According to the recommendations ${ }^{19}{ }^{20}$ of the American Heart Association, the average BP of the three nearest recordings was adopted for analysis. Adults with hypertension were defined as (1) systolic BP (SBP) $\geq 140 \mathrm{~mm} \mathrm{Hg}$ or diastolic BP (DBP) $\geq 90 \mathrm{~mm} \mathrm{Hg}$ or (2) currently taking antihypertensive medications. Accordingly, four categories or proportions of participants were defined as follows:

- Prevalence of hypertension: adults with hypertension (BP $\geq 140 / 90$ or currently taking antihypertensive medications) among the enrolled participants.

- Awareness of hypertension: adults with a self-reported diagnosis of hypertension by a physician among participants with hypertension.

- Treatment of hypertension: adults who currently take antihypertensive medication among participants with awareness of hypertension.

- Control of hypertension: adults who had attained an average $\mathrm{BP}$ of $<140 / 90 \mathrm{~mm} \mathrm{Hg}$ among participants by taking antihypertensive medication

\section{Measures of the SES}

Two domains of the iSES were measured in the survey: personal income and education. Individual monthly income was ascertained by a formal questionnaire (with New Taiwan dollar (NT\$) being converted into US dollar (US\$)), and it was categorised as 27 levels: no income, <US $\$ 170$, US $\$ 170-U S \$ 339$, US $\$ 340-U S \$ 682$, US $\$ 683-U S \$ 1024, \quad$ US $\$ 1025-U S \$ 1365$, US $\$ 1366$ US $\$ 1707$, US $\$ 1708-U S \$ 2049$, US $\$ 2050-U S \$ 2391$, US\$2392-US\$2732, US\$2733-US\$3074, US\$3075US\$3415, US\$3416-US\$3757, US\$3758-US\$4099, US\$3758-US\$4099, US\$4100-US\$4440, US\$4101US $\$ 4782, \quad$ US $\$ 4783-U S \$ 5124, \quad$ US $\$ 5125-U S \$ 5465$, US $\$ 5466-U S \$ 6148, \quad$ US $\$ 5466-U S \$ 6148, \quad$ US $\$ 6149$ 68US $\$ 31$ ， US $\$ 6832-U S \$ 8532, \quad$ US $\$ 8533-U S \$ 10,238$, US $\$ 10$ 239-US $\$ 11,956$, US $\$ 11$ 957-US $\$ 13,664$, US $\$ 11$ $957-\mathrm{US} \$ 17,080$, US $\$ 17$ 081-US $\$ 34,126$, and > US $\$ 34$ 126 during 2013-2016. Individual educational attainment was established and categorised as no education $(0$ years), less than elementary school (4 years), elementary school graduate (6 years), junior high school (8 years), junior high school graduate (9 years), less than senior high or vocational school (11 years), senior high or vocational school graduate (12 years), less than college (14 years), college graduate (16 years) and graduate school or higher (20 years). Thereafter, iSES was defined using a total score that was the sum of the $\mathrm{z}$ scores of income and education. Accordingly, the iSES was classified into three classes. From the lowest to the highest iSES classes, these tertiles were designated T1, T2 and T3, respectively.

\section{Statistical Analysis}

SAS software (version V.9.4; SAS Institute, Cary, North Carolina) was used for all statistical analyses. Continuous 
and discrete variables were expressed as the mean $\pm \mathrm{SD}$ and number $(n)$ and percentages, and they were analysed with Student's t test. Categorical variables were reported as proportions and were examined with a $\chi^{2}$ test or Fisher's exact test. The STDIZE (standardize) procedure (setting the method of standardisation (std), location of mean and scale of SD) was performed to obtain individual z-scores of personal income and educational level. Z-score transformation is the process of converting a raw continuous number into a standard score. The formula of the z-score is $(x-\mu) / \sigma$, where $x$ stands for a raw score, $\mu$ is the mean of the population and $\sigma$ is the SD of the population. The method of employing summing z-scores by combining different SESs was developed by Diez Roux and colleagues in their research. ${ }^{21}{ }^{22}$ A summary iSES z-score was constructed by summing z-scores of the two variables, individual income and education. The increasing values of z-scores signify an increasing socioeconomic advantage; therefore, participants with iSES z-scores of $<33$ rd percentile, 33rd percentile $\leq$ iSES z-scores $\leq 66$ th percentiles, and iSES z-scores of $>66$ th percentiles were categorised into tertiles of T1, T2 and T3, respectively. Simple logistic regression models were employed to obtain the OR and 95\% CI, with dependent variables being awareness, treatment and control of hypertension, and independent variables of three levels of iSES (the lowest iSES tertile of T1 was used as the reference group). In addition, stratification by age of 65 years was used for analysis. Applying the Bonferroni correction, a two-tailed $p$ value of $<0.0167$ was considered statistically significant. This $\mathrm{p}$ value was obtained by the Bonferroni correction formula $p$ value $\leq \alpha / \mathrm{m}$, where $\alpha$ is the desired overall alpha level and $\mathrm{m}$ is the number of hypotheses. In our study, we defined $\alpha$ as 0.05 and comparison of the three subgroups (or hypothesis) was conducted accordingly. Statistical power $(1-\beta)$ was estimated by specifying an $\alpha$ error of 0.05 , with the number of participants in each tertile of T1, T2 and T3, and with a probability of T1 as the reference group. STATA software (V.17; StataCorp, College Station, Texas) was used to calculate the statistical power $(1-\beta)$.

\section{Sensitivity analysis}

Participants who gave consent and completed phlebotomy for all laboratory testing of serum creatinine, glucose and HbAlc were included in this sensitivity analysis. In addition, the definition as given by the Eighth Joint National Committee ${ }^{23}$ was used as another definition of controlled hypertension. In the JNC8 ${ }^{23}$ standard, patients with a comorbidity of DM or CKD were asked to control their BP to $<140 / 90 \mathrm{~mm} \mathrm{Hg}$ at all ages, while older adults $>60$ years with no cardiovascular risk factors were given a goal of $<150 / 90 \mathrm{~mm} \mathrm{Hg}$. Multivariable logistic regression models were employed to obtain the OR and $95 \%$ CI, with dependent variables being awareness, treatment and control of hypertension and independent variables being three levels of iSES (the lowest iSES tertile of T1 was used as the reference group) and covariates of sex (man vs woman), dwelling (urban vs rural), smoking habit (current, former and never smokers), DM and CKD. The interaction terms among the iSES, age, and DM were accordingly examined.

\section{RESULTS}

\section{Population Characteristics}

In our study population of 4599 participants, the mean age was $53.2 \pm 18.5$ years, and $2306(50.1 \%)$ participants were women (online supplemental table S1). The mean SBP was $125.12 \pm 19.3 \mathrm{~mm} \mathrm{Hg}$, and DBP was $75.6 \pm 11.0$ $\mathrm{mm} \mathrm{Hg}$. Of the study cohort, 2636 participants agreed to undergo phlebotomy for fasting glucose and HbAlc, and 2647 agreed to undergo a creatinine test. Based on laboratory test results, we inferred that the prevalence of DM was $17.9 \%$, in accordance with the standard of the American Diabetic Association. ${ }^{12}$ Most participants had healthy renal function, and the proportions of CKD stages $0-1$ and stage 2 were $67.3 \%$ and $25.6 \%$, respectively, according to the CKD-EPI formula. The characteristics of the participants, who were stratified by the age of 65 years (table 1) and by three classes of iSES (online supplemental table S2), are shown accordingly. Additionally, the 33rd and 66th percentiles of iSES z-scores were-1.167 and 0.833 ; therefore, participants with z-scores of $<-1.167$, $1.167 \leq z$-scores $\leq 0.83$ and $z$-scores of $>0.8333$ were categorised as T1, T2 and T3, respectively. The numbers of participants in the three tertiles were 1489, 1621 and 1489 , respectively. Among the younger $(<65$ years $)$ and older ( $\geq 65$ years) groups, fewer older adults had incomes of US\$5000- US $\$ 10000$ and >US\$10 000, and most of the older adults had educational attainment of less than high school. The iSES z-scores for the younger and older groups were $0.50 \pm 2.33$ and $-1.86 \pm 1.95$, respectively. Older participants had fewer never smokers and more former smokers. Otherwise, the two age groups exhibited a similar distribution of dwelling in the metropolitan and township areas and geographic areas in Taiwan.

\section{General hypertension status}

The prevalence, awareness and medical treatment of hypertension in (1) four geographical areas and (2) different age and sex groups in Taiwan are shown in online supplemental table S3 and S4, respectively. Each geographical area (northern, central, southern and eastern areas) had similar prevalence, awareness levels and medical treatments. The inferred prevalence of hypertension in all enrolled participants was $37.0 \%$, while it was $39.9 \%$ for men and $34.2 \%$ for women. In general, $67.2 \%$ of hypertensive participants were aware of hypertension, and women $(72.3 \%)$ had a higher awareness of hypertension than men $(62.8 \%)$. Of those participants who were aware of their hypertension, $65.0 \%$ had received medical treatment, and women $(71.1 \%)$ were more willing to receive medical treatment than men $(59.9 \%)$. In addition, the proportions of the prevalence (figure 1), awareness (figure 2) and medical treatment 
Table 1 Characteristics of participants of the Nutrition and Health Survey in Taiwan by SES and age

\begin{tabular}{|c|c|c|c|}
\hline Participants & Younger ( $<65$ years) & Older ( $\geq 65$ years) & $P$ value \\
\hline $\mathrm{N}$ & 3076 & 1523 & \\
\hline Female sex, $n(\%)$ & $50.3 \%(1548 / 3076)$ & $49.8 \%(758 / 1523)$ & 0.7232 \\
\hline Annual income US\$, $n(\%)$ & & & $<0.0001^{*}$ \\
\hline$<$ US\$5000 & $30.3 \%(933 / 3076)$ & $67.6 \%(1029 / 1523)$ & \\
\hline \$US5000-US10 000 & $30.2 \%(928 / 3076)$ & $19.6 \%(298 / 1523)$ & \\
\hline$>$ US\$10 000 & $39.5 \%(1215 / 3076)$ & $12.9 \%(196 / 1523)$ & \\
\hline Educational attainment, $n(\%)$ & & & $<0.0001^{*}$ \\
\hline Less than high school & $39.1 \%(1202 / 3076)$ & $63.1 \%(961 / 1523)$ & \\
\hline High school graduate & $36.1 \%(1111 / 3076)$ & $25.7 \%(391 / 1523)$ & \\
\hline College or greater & $24.8 \%(763 / 3076)$ & $11.2 \%(171 / 1523)$ & \\
\hline$z$-score of iSES $\dagger$ & $0.50 \pm 2.33$ & $-1.86 \pm 1.95$ & \\
\hline iSES classes & & & $<0.0001^{*}$ \\
\hline Lowest tertile (T1) & $18.2 \%(561 / 3076)$ & $60.9 \%(928 / 1523)$ & \\
\hline Middle tertile (T2) & $39.1 \%(1204 / 3076)$ & $27.4 \%(417 / 1523)$ & \\
\hline Highest tertile (T3) & $42.6 \%(1311 / 3076)$ & $11.7 \%(178 / 1523)$ & \\
\hline Smoking status $\ddagger$ & & & $<0.0001^{*}$ \\
\hline Current & $65.6 \%(1935 / 2948)$ & $67.7 \%(1002 / 1480)$ & \\
\hline Former & $14.7 \%(432 / 2948)$ & $22.6 \%(335 / 1480)$ & \\
\hline Never & $19.7 \%(581 / 2948)$ & $9.7 \%(143 / 1480)$ & \\
\hline Dwelling & & & 0.7243 \\
\hline Metropolitan area & $26.0 \%(799 / 3076)$ & $26.5 \%(403 / 1523)$ & \\
\hline Township & $74.0 \%(2277 / 3076)$ & $73.5 \%(1120 / 1523)$ & \\
\hline Geographical region & & & 0.9139 \\
\hline Northern & $24.0 \%(738 / 3076)$ & $24.4 \%(371 / 1523)$ & \\
\hline Central & $9.8 \%(302 / 3076)$ & $10.4 \%(158 / 1523)$ & \\
\hline Southern & $35.6 \%(1095 / 3076)$ & $34.9 \%(532 / 1523)$ & \\
\hline Eastern & $30.6 \%(941 / 3076)$ & $30.3 \%(462 / 1523)$ & \\
\hline
\end{tabular}

*Statistical significance with a $\mathrm{p}$ value $<0.05$.

†The $z$-score of the iSES was constructed by summing the $z$-scores of individual annual income and education years. $\ddagger$ There were 171 missing observations

iSES, individual socioeconomic score; SES, socioeconomic status.

(figure 3) increased with age for both female and male participants. The mean SBP increased for each group, while the greatest level of mean DBP occurred in the age groups of 50-59 years (figure 4 ). Using the JNC 8 criteria to categorise BP control, $60.3 \%$ of men and $63.6 \%$ of women who were receiving medical treatment had their BP controlled (online supplemental table S5). When using the cut-off of $140 / 90 \mathrm{~mm} \mathrm{Hg}$, slightly fewer people taking treatment had their BP controlled. BP levels were greater in those taking antihypertensive medications than in those who were not taking these agents (online supplemental table S6) .

The prevalence, awareness and medical treatment of hypertension according to the three classes of iSES are shown in table 2. The SBP and DBP in each age group and the iSES group are shown in online supplemental table S7. Younger participants in the $\mathrm{T} 1$ class had a higher prevalence of hypertension (33.3\%) than those in T2 $(22.1 \%)$ and T3 $(21.8 \%) \quad(\mathrm{p}<0.0001)$. Younger participants in $\mathrm{T} 1$ paradoxically had a higher proportion of awareness $(66.3 \%)$ than those in T2 $(56.0 \%)$ and T3 $(54.4 \%)$. All iSES groups of older participants showed a similar prevalence of hypertension $(\mathrm{p}=0.3499)$. Older participants in all tertiles showed similar levels of awareness. For medical treatment, younger and older participants showed no significant difference in any of the iSES groups.

Factors associated with the hypertension status (by a univariable analysis)

The results of the univariate analysis between iSES and hypertension status are shown in table 3. Compared with T1, higher iSES was associated with decreased awareness of hypertension at all ages (OR of T2: $0.43,95 \%$ CI 0.37 to 0.51 , 


\section{Prevalence of Hypertension in Different Age Groups}

$80.00 \%$

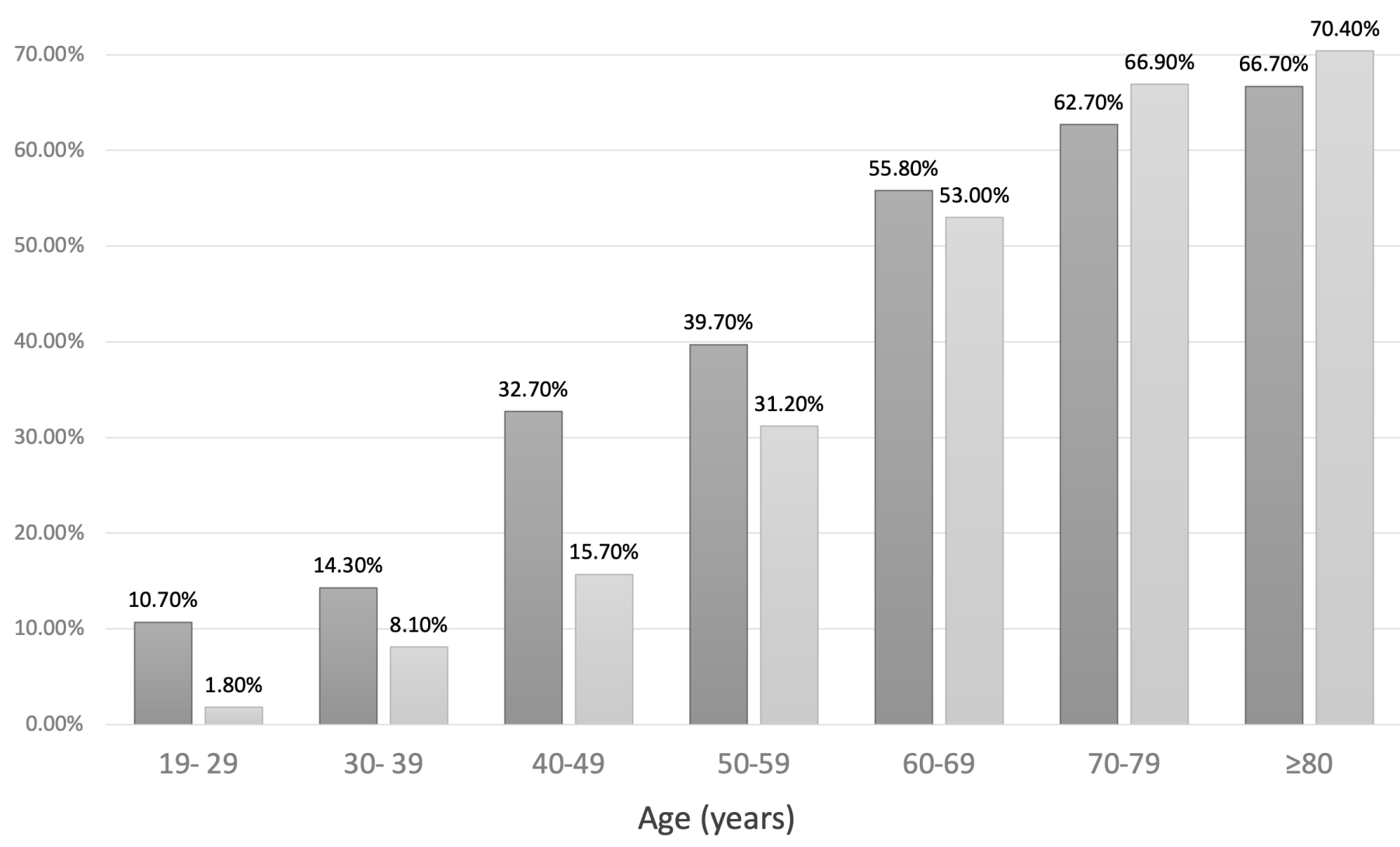

Male $\square$ Female

Figure 1 Prevalence of hypertension in different age groups. The denominators for age groups of 19-29, 30-39, 40-49, 50-59, 60-69, 70-79 and $\geq 80$ for men were 337, 286, 312, 398, 448, 359 and 153, and for women were 336, 298, 306, 414, 438, 362 and 152, respectively. The numbers constitute the full sample of men and women separately.

$\mathrm{p}<0.0001$; OR of T3: $0.30,95 \%$ CI 0.25 to $0.36, \mathrm{p}<0.0001)$ and younger participants (OR of T2: $0.50,95 \%$ CI 0.39 to 0.64 , $\mathrm{p}<0.0001$; OR of T3: $0.48,95 \%$ CI 0.37 to $0.61, \mathrm{p}<0.0001)$. In addition, a higher iSES was negatively associated with medical treatment at all ages (OR of T2: $0.45,95 \%$ CI 0.39 to 0.53 , $\mathrm{p}<0.0001$; OR of T3: $0.28,95 \%$ CI 0.23 to $0.33, \mathrm{p}<0.0001)$ and younger participants (OR of T2: $0.57,95 \%$ CI 0.44 to 0.75 , $\mathrm{p}<0.0001$; OR of T3: $0.49,95 \%$ CI 0.37 to $0.64, \mathrm{p}<0.0001)$. In contrast, a higher iSES was associated with better control of hypertension of $\mathrm{BP}<140 / 90 \mathrm{~mm} \mathrm{Hg}$ for all ages (OR of T2: $1.80,95 \%$ CI 1.53 to $2.12, \mathrm{p}<0.0001$; OR of T3: $2.03,95 \%$ CI 1.71 to $2.41, \mathrm{p}<0.0001)$ and younger participants (OR of T2: $1.74,95 \%$ CI 1.35 to 2.24 , p $<0.0001$; OR of T3: $1.52,95 \%$ CI 1.19 to $1.94, \mathrm{p}=0.0009)$. For older participants, the iSES was not associated with awareness, treatment or control of hypertension. The statistical power $(1-\beta)$ for each tertile comparison was adequate (online supplemental table $\mathrm{S} 8$ and figure $\mathrm{S} 1)$.

Sensitivity analysis: factors associated with the hypertension status (by a multivariable Analysis)

The results of the multivariable analysis between iSES and hypertension status are shown in figures 5 and 6 and online supplemental table S9. Similar to that in the univariable analysis, a higher iSES was negatively associated with awareness and treatment for hypertension, and positively associated with better control of hypertension, except in older participants. In addition, the covariate of male sex was associated with poor control of hypertension in terms of JNC8 criteria (OR: $0.70,95 \%$ CI 0.55 to 0.88 , $\mathrm{p}=0.0021$ ) and control of BP to $<140 / 90 \mathrm{~mm} \mathrm{Hg}$ (OR: $0.64,95 \%$ CI 0.50 to $0.83, \mathrm{p}=0.0006)$. Current and previous smokers were associated with increased awareness (OR: 1.50, 95\% CI 1.08 to $2.09, \mathrm{p}=0.0152$ for previous smokers; OR: 1.70 , $95 \%$ CI 1.19 to $2.44, \mathrm{p}=0.0036$ for current smokers) and medical treatment for hypertension (OR: $1.47,95 \% \mathrm{CI}$ 1.05 to $2.06, \mathrm{p}=0.0241$ for previous smokers; OR: 1.53 , $95 \%$ CI 1.06 to $2.21, p=0.0241$ for current smokers), but this association only existed for younger current smokers after stratification. Diagnosis of DM and CKD was associated with a threefold increase in awareness and medical treatment for hypertension. Nevertheless, participants with DM and CKD were associated with a greater difficulty in controlling hypertension. The magnitude of the association between DM and CKD was more accentuated 


\section{Awareness of Hypertension in Different Age Groups}

$90.00 \%$

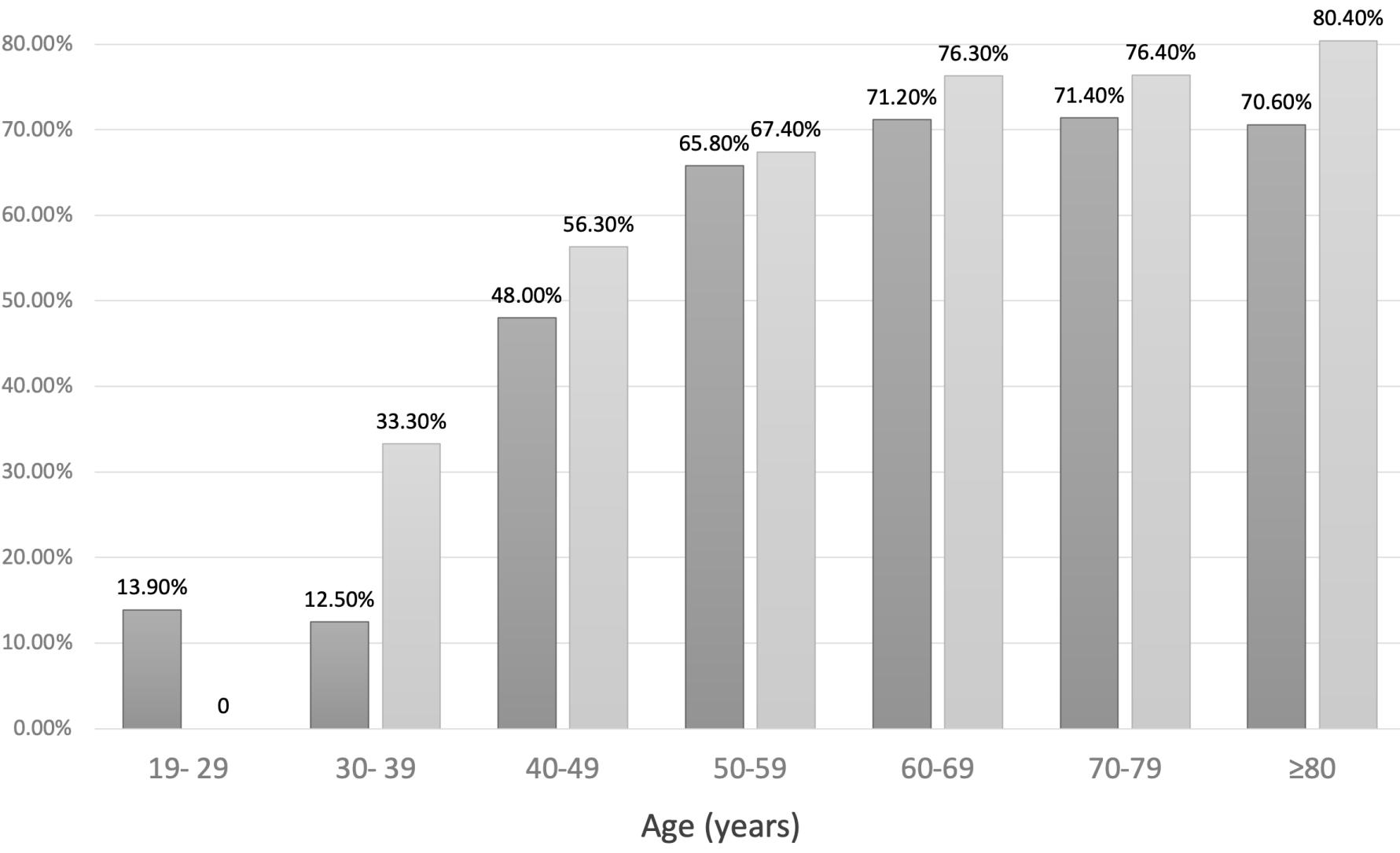

\section{Male $\square$ Female}

Figure 2 Awareness of hypertension in different age groups. The denominators for age groups of 19-29, 30-39, 40-49, 50-59, 60-69, 70-79 and $\geq 80$ for men were 36, 40, 102, 158, 250, 224 and 102, and for women were 6, 24, 48, 129, 232, 242 and 107, respectively. The numbers constitute the full sample of men and women separately.

in younger participants. The interaction terms among continuous iSES, age, DM and CKD are shown in online supplemental table S10, and the only significant interaction term was between iSES and age.

\section{DISCUSSION}

The most significant finding in this study was that younger participants with a higher iSES had scored less in awareness and treatment of hypertension, and fewer of them were undergoing treatment for this disease. However, a higher iSES score was associated with better hypertension control. In addition, current smokers and subjects known to have DM and CKD were robustly associated with positive awareness and treatment attitudes about hypertension despite having greater difficulty controlling their BP.

In Taiwan, the NHI was introduced in 1995 and is characterised by great accessibility and short waiting times when visiting physicians. ${ }^{24}$ With its comprehensive coverage of all medical costs of residents for treating hypertension and hypertension-related diseases, ${ }^{24}$ the present study provides insights regarding how to increase awareness of and treatment for hypertension in Taiwan.
Compared with the NAHSIT 1993-1996, ${ }^{1}$ this new survey showed that the urban-rural gaps in awareness, treatment and control of hypertension had diminished in Taiwan. However, there was a gap in awareness and treatment of hypertension between the younger and older participants.

The primary step for good management of hypertension is to make people aware of hypertension. ${ }^{25-28}$ In most cases, awareness of hypertension is probably rooted in a clinical diagnosis of hypertension by a physician. ${ }^{28}$ Second, knowledge of hypertension is the driving force to seek medical resources and to maintain routine health checks. ${ }^{29}{ }^{30}$ In our study, despite the younger group with a higher iSES having a lower awareness and rate of treatment, these participants were actually controlling their hypertension well. A prior analysis from the NHI database in Taiwan concluded that patients with higher education levels are likely to obtain multiple sources of health care ${ }^{30}$ Recent studies have indicated that knowledge of hypertension is independent, ${ }^{31} 32$ and it acts in an additive manner ${ }^{29}$ to improve control of hypertension. Patients with a higher iSES have more positive 


\section{Treatment of Hypertension in Different Age Groups}

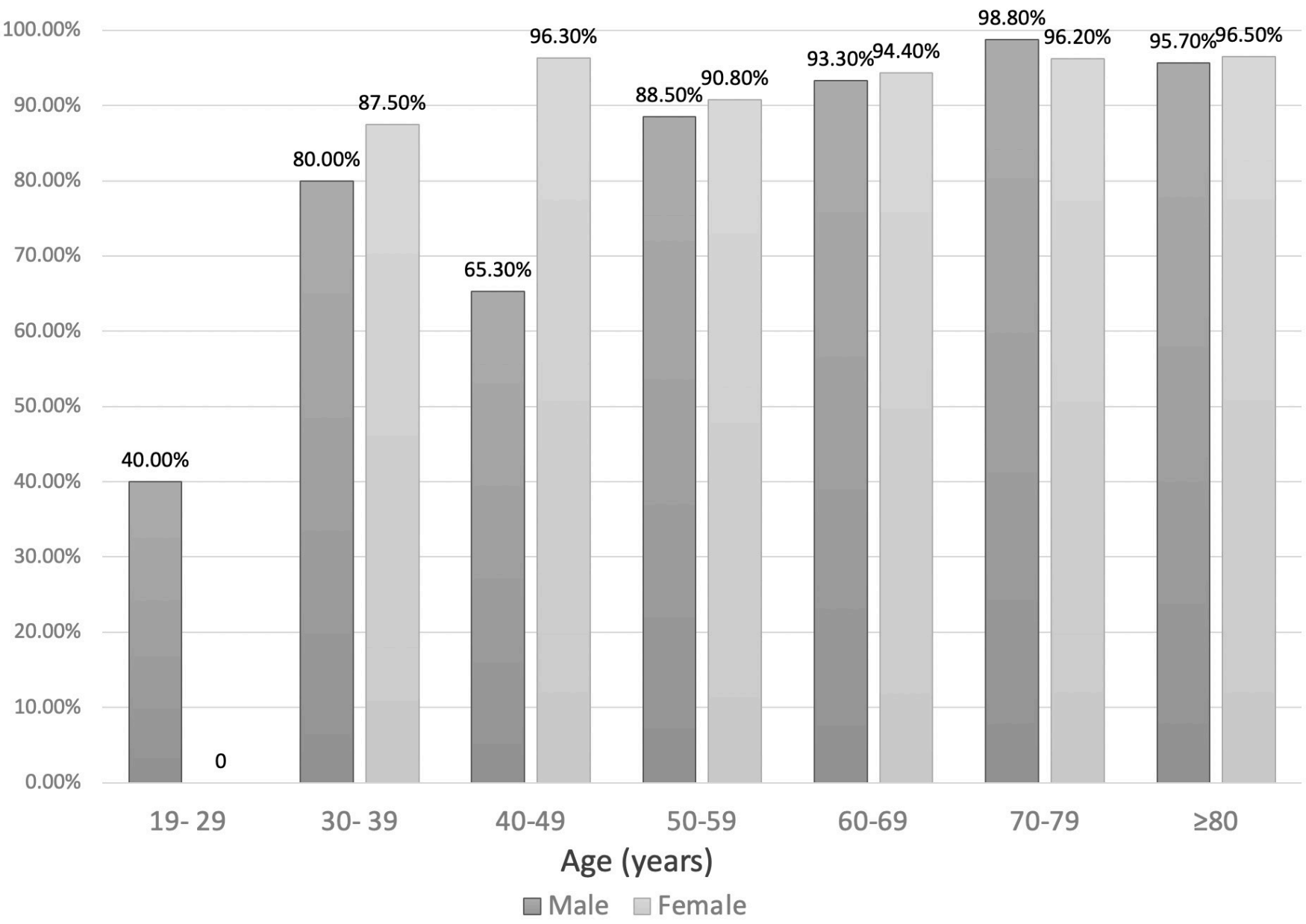

Figure 3 Treatment of hypertension in different age groups. The denominators for age groups of 19-29, 30-39, 40-49, 50-59, 60-69, 70-79 and $\geq 80$ for men were 36, 41, 102, 158, 250, 225 and 102, and for women were 6, 24, 48, 129, 232, 242 and 107, respectively. The numbers constitute the full sample of men and women separately.

attitudes and stronger motivation to adhere to healthy lifestyle. ${ }^{33}$ Our results suggested that education about increasing awareness of hypertension should focus on individuals in the younger age group in Taiwan. We deduced that younger adults may easily ignore their BP status because they think that hypertension is a disease that affects older adults. Once the state of hypertension

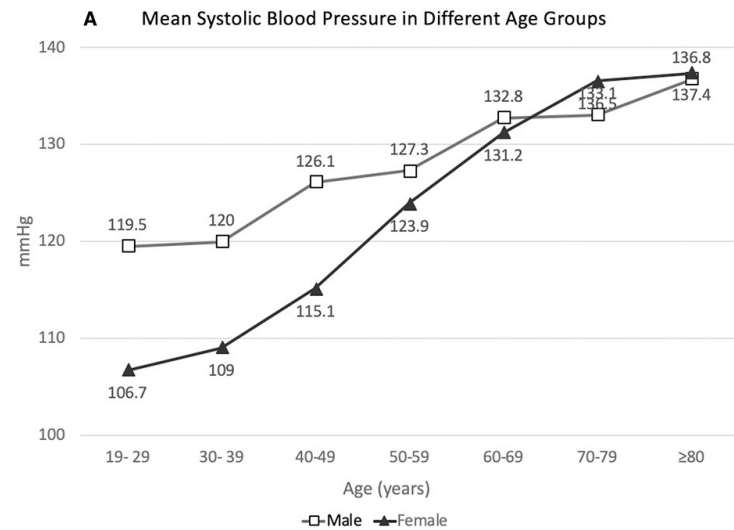

B Mean Diastolic Blood Pressure in Different Age Groups

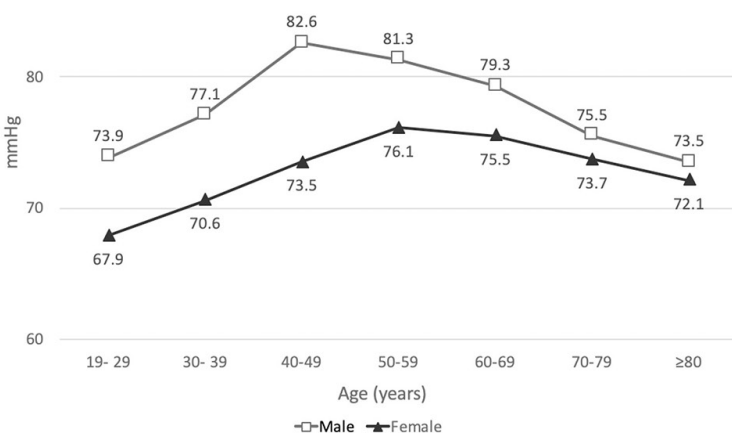

Figure 4 (A) Mean systolic blood pressure and (B) mean diastolic blood pressure in different age groups. The denominators for age groups of 19-29, 30-39, 40-49, 50-59, 60-69, 70-79 and $\geq 80$ for men were 337, 286, 312, 398, 448, 359 and 153 , and for women were $336,298,306,414,438,362$ and 152, respectively. The numbers constitute the full sample of men and women separately. 


\begin{tabular}{|c|c|c|c|c|}
\hline iSES class & Lowest tertile (T1) & Middle tertile (T2) & Highest tertile (T3) & P value \\
\hline \multicolumn{5}{|l|}{ All ages $(\mathrm{N}=4599)$} \\
\hline Prevalence of hypertension & $52.7 \%(785 / 1489)$ & $32.5 \%(526 / 1621)$ & $26.3 \%(391 / 1489)$ & $<0.0001^{*}$ \\
\hline Awareness of hypertension & $72.5 \%(568 / 784)$ & $65.1 \%(342 / 525)$ & $59.5 \%(232 / 390)$ & $<0.0001^{*}$ \\
\hline Treatment of hypertension & $93.8 \%(533 / 568)$ & $94.7 \%(324 / 342)$ & $88.4 \%(205 / 232)$ & $0.0072^{*}$ \\
\hline \multicolumn{5}{|l|}{$<65$ years $(\mathrm{N}=3076)$} \\
\hline Prevalence of hypertension & $33.3 \%(187 / 561)$ & $22.1 \%(266 / 1204)$ & $21.8 \%(286 / 1311)$ & $<0.0001^{*}$ \\
\hline Awareness of hypertension & $66.3 \%(124 / 187)$ & $56.0 \%(149 / 266)$ & $54.4 \%(155 / 285)$ & $0.0265^{*}$ \\
\hline Treatment of hypertension & $84.7 \%(105 / 124)$ & $92.6 \%(138 / 149)$ & $84.5 \%(131 / 155)$ & 0.0584 \\
\hline \multicolumn{5}{|l|}{$\geq 65$ years $(\mathrm{N}=1523)$} \\
\hline Prevalence of hypertension & $64.4 \%(598 / 928)$ & $62.4 \%(260 / 417)$ & $59.0 \%(105 / 178)$ & 0.3499 \\
\hline Awareness of hypertension & $74.4 \%(444 / 597)$ & $74.5 \%(193 / 259)$ & $73.3 \%(77 / 105)$ & 0.9707 \\
\hline Treatment of hypertension & $96.4 \%(428 / 444)$ & $96.4 \%(186 / 193)$ & $96.1 \%(74 / 77)$ & 0.9920 \\
\hline
\end{tabular}

*Statistical significance with Bonferroni correction $p<0.0167$.

iSES, individual socioeconomic status;

was recognised, the younger high-iSES group had better medical compliance.

For older participants, iSES states were completely dissociated from the awareness, treatment and control states of hypertension. This could be attributed to public recognition of the high prevalence of hypertension in the elderly. In addition, older participants were more likely to have other illnesses or chronic diseases, and they probably visited clinics or hospitals more often. With NHI coverage, older participants in all iSES classes had equal medical availability. Physicians and participants themselves had more chances to recognise their BP status at each clinic or hospital visit. Knowing that one had DM or CKD was an important factor associated with awareness and treatment of hypertension in our analyses. To our understanding, this finding directly confirms that participants with chronic illnesses had a higher awareness and were more willing to receive medical treatment, and they indirectly supported the positive relationship between visits to physicians and increased awareness and treatment of hypertension. Most health education has focused on older adults in the last decade, or toward an aged society in Taiwan. ${ }^{18}$ Conversely, our study suggests that health education for the younger group cannot be neglected.

The strengths of this survey are as follows. First, this nationwide sample included a large number of representative participants and displayed adequate statistical power. Second, we adopted two definitions for controlling

Table 3 Univariable analysis of the association between iSES and awareness, treatment, control of hypertension

\begin{tabular}{|c|c|c|c|c|c|c|}
\hline \multirow[t]{2}{*}{ iSES class } & \multicolumn{2}{|l|}{ Awareness } & \multicolumn{2}{|l|}{ Treatment } & \multicolumn{2}{|c|}{ Controlled<140/90 mm Hg } \\
\hline & OR $(95 \% \mathrm{Cl})$ & $p$ value & OR $(95 \% \mathrm{Cl})$ & p value & OR $(95 \% \mathrm{Cl})$ & p value \\
\hline \multicolumn{7}{|l|}{ All ages } \\
\hline Middle tertile (T2) & $0.43(0.37$ to 0.51$)$ & $<0.0001$ & 0.45 (0.39 to 0.53$)$ & $<0.0001$ & 1.80 (1.53 to 2.12$)$ & $<0.0001$ \\
\hline High tertile (T3) & $0.30(0.25$ to 0.36$)$ & $<0.0001$ & 0.28 (0.23 to 0.33$)$ & $<0.0001$ & 2.03 (1.71 to 2.41$)$ & $<0.0001$ \\
\hline \multicolumn{7}{|l|}{$<65$ years } \\
\hline Lower tertile (T1) & Ref. & & Ref. & & Ref. & \\
\hline Middle tertile (T2) & 0.50 (0.39 to 0.64$)$ & $<0.0001^{*}$ & $0.57(0.44$ to 0.75$)$ & $<0.0001^{*}$ & 1.74 (1.35 to 2.24$)$ & $<0.0001^{*}$ \\
\hline High tertile (T3) & $0.48(0.37$ to 0.61$)$ & $<0.0001^{*}$ & $0.49(0.37$ to 0.64$)$ & $<0.0001^{*}$ & 1.52 (1.19 to 1.94$)$ & $0.0009^{*}$ \\
\hline \multicolumn{7}{|l|}{$\geq 65$ years } \\
\hline Lower tertile (T1) & Ref. & & Ref. & & Ref. & \\
\hline
\end{tabular}

*Statistical significance with Bonferroni correction $\mathrm{p}<0.0167$.

iSES, individual socioeconomic status.; 

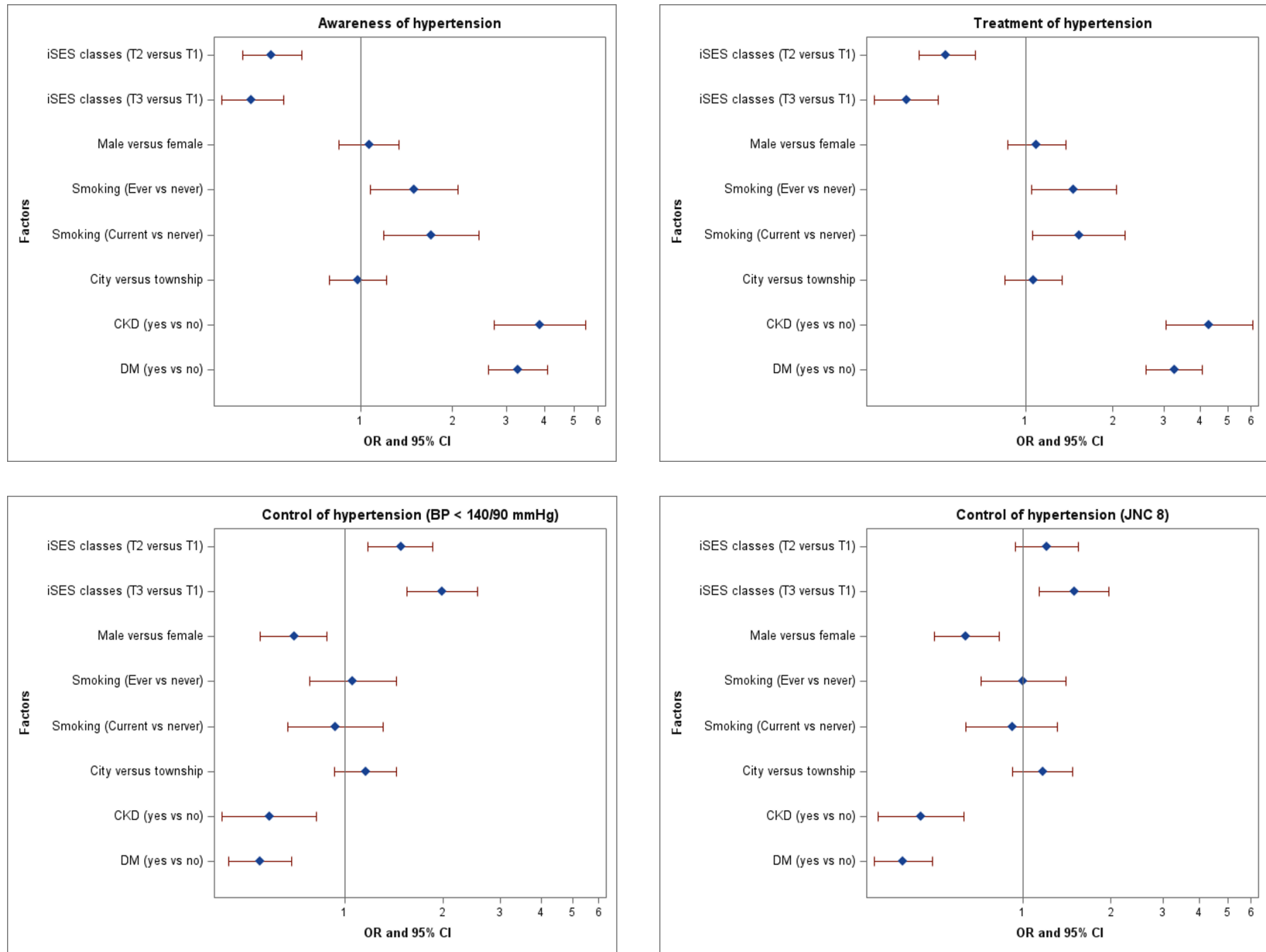

Figure 5 Multivariable analysis of the factors associated with awareness, treatment and control of hypertension (total population). In the multivariable analysis, each factor was adjusted for the other factors. BP, blood pressure; CKD, chronic kidney disease; DM, diabetes mellitus; iSES, individual socioeconomic status; JNC8, Eighth Joint National Committee.

hypertension with (1) $\mathrm{BP}<140 / 90 \mathrm{~mm} \mathrm{Hg}$ and (2) JNC8. ${ }^{23}$ The reasons we used two definitions were (1) to comply with the standards of most published studies with simple criteria of $140 / 90 \mathrm{~mm} \mathrm{Hg}$ or current therapy with antihypertensive medications and (2) to comply with the treatment guideline ${ }^{34}$ published by the Taiwan Society of Cardiology in 2015. During the study period, 20132016, most physicians in Taiwan followed the treatment guideline $^{34}$ of the Taiwan Society of Cardiology, which was adapted from JNC8. Therefore, a healthy population of $\geq 60$-year-old without diabetes and CKD was treated with a BP goal of $<150 / 90 \mathrm{~mm} \mathrm{Hg}$, while the others had a BP goal of $<140 / 90 \mathrm{~mm} \mathrm{Hg}$. In our analyses, a higher SES was associated with better control of hypertension in both the JNC8 and 140/90 mm Hg criteria, but this relationship was not observed in older adults. In contrast, $\mathrm{DM}$ and CKD were robustly associated with unfavourable control of hypertension in both the younger and older age groups. BP control was highly associated with the disease state and medical comorbidities. Consequently, these findings suggest that improving awareness and medical treatment should enhance hypertension control in the younger group with a higher iSES, but not in the older group, due to their already existing high level of awareness.

This study has some limitations. It had a cross-sectional design in the form of NAHSIT. Causal associations between SESs and hypertension could not be inferred from our survey. The importance of this survey was to guide us in what to do to increase hypertension control with NHI coverage of all personal medical costs in Taiwan. Since the NAHIST 2013-2016 adopted a door-to-door survey, people who lived in extremely remote areas, including islands near the east coastline and very high-mountain regions, were not covered in our study. Additionally, foreigners living in Taiwan were not investigated in this study since our nationwide sampling was based on native census data. During our study analysis, we calculated the average $\mathrm{BP}$ of the three nearest recordings according to the recommendations ${ }^{19} 20$ of the American Heart Association. The WHO STEPwise approach to surveillance protocol suggests discarding the first BP reading and 

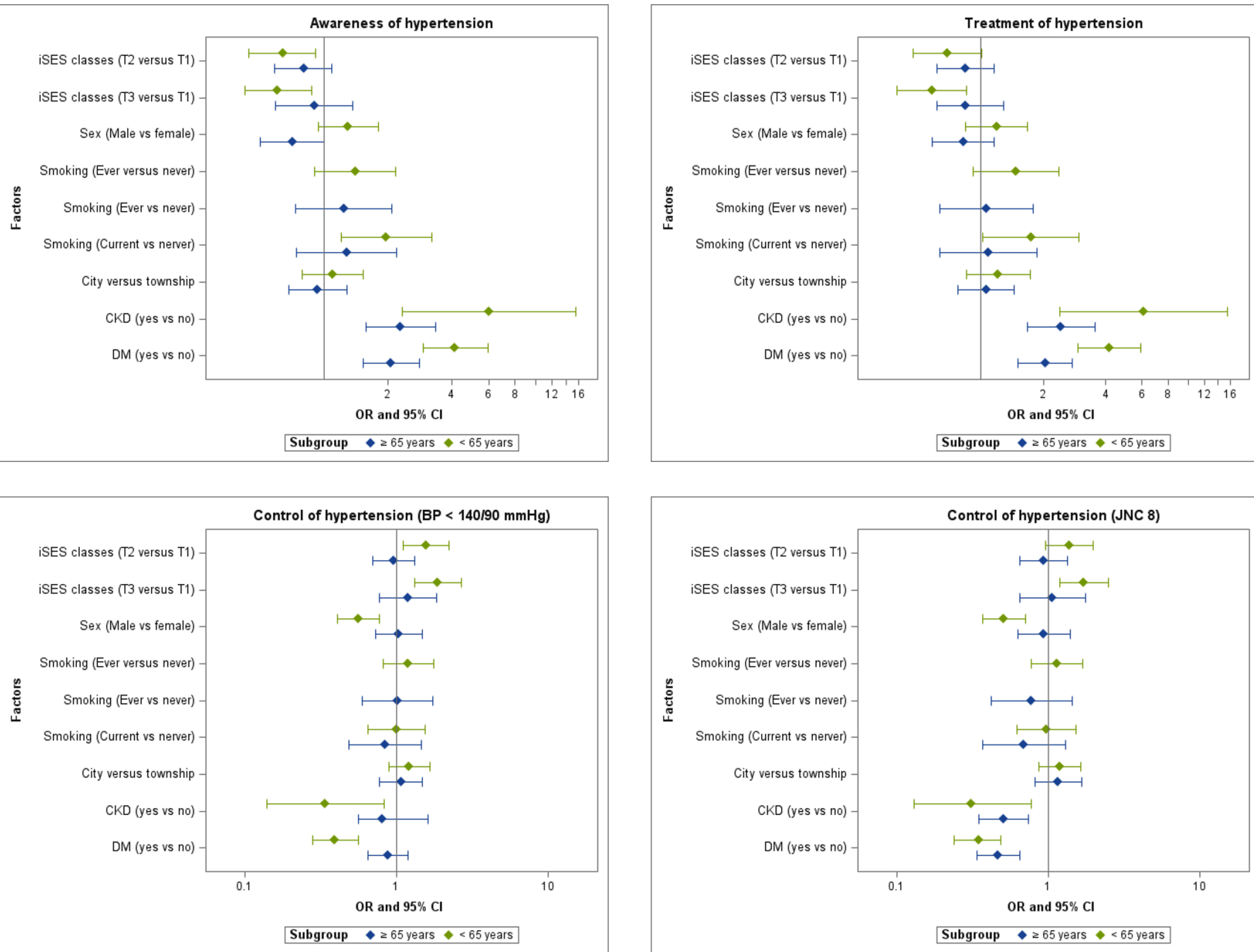

Figure 6 Multivariable analysis of the factors associated with awareness, treatment and control of hypertension in younger (<65 years) and older participants ( $\geq 65$ years). In the multivariable analysis, each factor was adjusted for the other factors. CKD, chronic kidney disease; DM, diabetes mellitus; iSES, individual socioeconomic status; JNC8, Eighth Joint National Committee.

taking the average $\mathrm{BP}$ of the last two readings. However, two studies investigated the effect of excluding first BP and found no significant impact on the distribution of categorising participants into two groups of BP $<140 / 90$ $\mathrm{mm} \mathrm{Hg}$ and $\mathrm{BP} \geq 140 / 90 \mathrm{~mm} \mathrm{Hg} .{ }^{3536}$ A recent large-scale study ${ }^{36}$ concluded that there was an extremely small difference (approximately $1.5 \mathrm{~mm} \mathrm{Hg}$ ) between excluding and including first BP measurement in the settings of random population sample, general practice office and specialised cardiovascular centres. Despite the small difference between our study and the others, we tended to keep the first readings of $\mathrm{BP}$ because we consider that it reflects short-term variability in reality. Third, half of the healthy participants in our study agreed to undergo phlebotomy for laboratory testing. However, this missing information should not distort our main results because no blood testing was needed to measure the prevalence, awareness, treatment and control of hypertension. Additionally, the older and younger participants had similar proportions of completed phlebotomy for blood sugar and serum creatinine (online supplemental table S1)); therefore, this missing information should not cause differential estimation in younger and older age groups. Finally, NAHSIT 2013-2016 had no direct measurement of knowledge of hypertension.

In conclusion, strategies to improve awareness and treatment of hypertension should focus on younger patients with higher SESs. It is probable that the younger group may easily neglect personal healthcare. Further investigations into the causes of this phenomenon are, therefore, required in the future.

\section{Author affiliations}

${ }^{1}$ Department of Public Health, School of Medicine, College of Medicine, Taipei Medical University, Taipei, Taiwan

${ }^{2}$ School of Public Health, College of Public Health, Taipei Medical University, Taipei, Taiwan

${ }^{3}$ Department of Emergency Medicine, Taipei Medical University Hospital, Taipei, Taiwan

${ }^{4}$ Department of Critical Care Medicine, Taipei Medical University Hospital, Taipei, Taiwan

${ }^{5}$ Institute of Biomedical Science, Academia Sinica, Taipei, Taiwan

${ }^{6}$ Nutrition Research Center, Taipei Medical University Hospital, Taipei, Taiwan 
Contributors S-F L: conceptualisation, formal analysis, writing—original draft, writing —review and editing. T-T K: conceptualisation. W-H P: conceptualisation, investigation, supervision. C-H B: conceptualisation, data curation, formal analysis, funding acquisition, supervision.S-F L and C-H B are responsible for overall content as the guarantors.

Funding This work was funded by the Health Promotion Administration, Ministry of Health and Welfare (reference number: MOHW108-HPA-H-114-1 34 703, MOHW109-HPA-H-114-1 44 702), and by the Ministry of Science and Technology, Taiwan, in the form of a grant awarded to CHB (reference number: MOST 107-2314-B-038-072-MY3, MOST 110-2314-B038-056-MY3)

Competing interests None declared.

\section{Patient consent for publication Not applicable.}

Ethics Statement The Institutional Review Board of Biomedical Science Research, Academia Sinica (reference number: AS-IRB01-13067) and the Research Ethics Committee, National Health Research Institutes, Taiwan (reference number: EC1020110) approved this study, and informed consent was obtained from all the participants. Patients or the public were not involved in the design, conduct, reporting, or dissemination plans of our research

Provenance and peer review Not commissioned; externally peer reviewed.

Data availability statement Data may be obtained from a third party and are not publicly available. Data are only available from the formal proposal to the Health and Welfare Data Science Center, Ministry of Health and Welfare, Taiwan. Contact information of the Ministry of Health and Welfare is as follows: Address: No.488, Sec. 6, Zhongxiao E. Rd., Nangang Dist., Taipei City 115204, Taiwan (R.0.C.); Tel: (+886)2-8590-6666; Fax: (+886)2-8590-6000.

Supplemental material This content has been supplied by the author(s). It has not been vetted by BMJ Publishing Group Limited (BMJ) and may not have been peer-reviewed. Any opinions or recommendations discussed are solely those of the author(s) and are not endorsed by BMJ. BMJ disclaims all liability and responsibility arising from any reliance placed on the content. Where the content includes any translated material, BMJ does not warrant the accuracy and reliability of the translations (including but not limited to local regulations, clinical guidelines, terminology, drug names and drug dosages), and is not responsible for any error and/or omissions arising from translation and adaptation or otherwise.

Open access This is an open access article distributed in accordance with the Creative Commons Attribution Non Commercial (CC BY-NC 4.0) license, which permits others to distribute, remix, adapt, build upon this work non-commercially, and license their derivative works on different terms, provided the original work is properly cited, appropriate credit is given, any changes made indicated, and the use is non-commercial. See: http://creativecommons.org/licenses/by-nc/4.0/.

\section{ORCID iD}

Chyi Huey Bai http://orcid.org/0000-0002-4658-1088

\section{REFERENCES}

1 Pan WH, Chang HY, Yeh WT, et al. Prevalence, awareness, treatment and control of hypertension in Taiwan: results of nutrition and health survey in Taiwan (NAHSIT) 1993-1996. J Hum Hypertens 2001;15:793-8.

2 Su T-C, Bai C-H, Chang H-Y, et al. Evidence for improved control of hypertension in Taiwan: 1993-2002. J Hypertens 2008;26:600-6.

3 Satoh M, Ohkubo T, Asayama K, et al. Lifetime risk of stroke and coronary heart disease deaths according to blood pressure level: EPOCH-JAPAN (evidence for cardiovascular prevention from observational cohorts in Japan). Hypertension 2019;73:52-9.

4 Pistoia F, Sacco S, Degan D, et al. Hypertension and stroke: epidemiological aspects and clinical evaluation. High Blood Press Cardiovasc Prev 2016;23:9-18.

5 Roth GA, Johnson C, Abajobir A, et al. Global, regional, and national burden of cardiovascular diseases for 10 causes, 1990 to 2015. J Am Coll Cardiol 2017;70:1-25.

6 Hsiao A-J, Chen L-H, Lu T-H. Ten leading causes of death in Taiwan: a comparison of two grouping Lists. J Formos Med Assoc 2015;114:679-80.

7 Wu X, Wang Z. Role of socioeconomic status in hypertension among Chinese middle-aged and elderly individuals. Int $J$ Hypertens 2019;2019:1-6.

8 McDoom MM, Palta P, Vart P, et al. Late life socioeconomic status and hypertension in an aging cohort: the Atherosclerosis risk in Communities study. J Hypertens 2018;36:1382-90.
9 Leng B, Jin Y, Li G, et al. Socioeconomic status and hypertension: a meta-analysis. J Hypertens 2015;33:221-9.

10 Lin S-F, Fan Y-C, Chou C-C, et al. Body composition patterns among normal glycemic, pre-diabetic, diabetic health Chinese adults in community: NAHSIT 2013-2016. PLoS One 2020;15:e0241121.

11 Tu S-H, Chen C, Hsieh Y-T, et al. Design and sample characteristics of the 2005-2008 nutrition and health survey in Taiwan. Asia Pac J Clin Nutr 2011;20:225-37.

12 Association AD. 2 Classification and Diagnosis of Diabetes:Diabetes Care 2020;43:S14-31.

13 Levey AS, Stevens LA, Schmid CH, et al. A new equation to estimate glomerular filtration rate. Ann Intern Med 2009;150:604-12.

14 Sharma P, McCullough K, Scotland G, et al. Does stage-3 chronic kidney disease matter?: a systematic literature review. Br J Gen Pract 2010;60:e266-76.

15 Winearls CG, Glassock RJ. Dissecting and refining the staging of chronic kidney disease. Kidney Int 2009;75:1009-14.

16 Levey AS, Eckardt K-U, Tsukamoto Y, et al. Definition and classification of chronic kidney disease: a position statement from kidney disease: improving global outcomes (KDIGO). Kidney Int 2005;67:2089-100

$17 \mathrm{Hu}$ H-M. Facing an aging Society: Taiwan's universities in crisis. Gerontol Geriatr Educ 2020;41:233-41.

18 Lin Y-Y, Huang C-S. Aging in Taiwan: building a Society for active aging and aging in place. Gerontologist 2016;56:176-83.

19 Pickering TG, Hall JE, Appel LJ, et al. Recommendations for blood pressure measurement in humans and experimental animals: Part 1: blood pressure measurement in humans: a statement for professionals from the Subcommittee of professional and public education of the American heart association Council on high blood pressure research. Hypertension 2005;45:142-61.

20 Bakris GL. The implications of blood pressure measurement methods on treatment targets for blood pressure.. Circulation 2016;134:904-5.

21 Diez Roux AV, Merkin SS, Hannan P, et al. Area characteristics, individual-level socioeconomic indicators, and smoking in young adults: the coronary artery disease risk development in young adults study. Am J Epidemiol 2003;157:315-26.

22 Diez-Roux AV, Kiefe Cl, Jacobs DR, et al. Area characteristics and individual-level socioeconomic position indicators in three population-based epidemiologic studies. Ann Epidemiol 2001;11:395-405.

23 James PA, Oparil S, Carter BL. Evidence-Based guideline for the management of high blood pressure in adults: report from the panel members appointed to the eighth joint National Committee (JNC 8). JAMA 2014 2014;311:507-20.

$24 \mathrm{Wu}$ T-Y, Majeed A, Kuo KN. An overview of the healthcare system in Taiwan. London J Prim Care 2010;3:115-9.

25 Glynn LG, Murphy AW, Smith SM, et al. Self-monitoring and other non-pharmacological interventions to improve the management of hypertension in primary care: a systematic review. Br J Gen Pract 2010;60:e476-88.

26 Alam DS, Chowdhury MAH, Siddiquee AT, et al. Awareness and control of hypertension in Bangladesh: follow-up of a hypertensive cohort. BMJ Open 2014;4:e004983.

27 Jones C, Simpson SH, Mitchell D, et al. Enhancing hypertension awareness and management in the elderly: lessons learned from the Airdrie Community Hypertension Awareness and Management Program (A-CHAMP). Can J Cardiol 2008;24:561-7.

28 Mills KT, Bundy JD, Kelly TN, et al. Global disparities of hypertension prevalence and control: a systematic analysis of population-based studies from 90 countries. Circulation 2016;134:441-50.

29 Ragavan RS, Joshi R, Evans RG, et al. Additive association of knowledge and awareness on control of hypertension: a crosssectional survey in rural India. J Hypertens 2021;39:107-16.

30 Sanne S, Muntner P, Kawasaki L, et al. Hypertension knowledge among patients from an urban clinic. Ethn Dis 2008;18:42-7.

31 Akoko BM, Fon PN, Ngu RC, et al. Knowledge of hypertension and compliance with therapy among hypertensive patients in the bamenda health district of Cameroon: a cross-sectional study. Cardiol Ther 2017;6:53-67.

32 Ozoemena EL, Iweama CN, Agbaje OS, et al. Effects of a health education intervention on hypertension-related knowledge, prevention and self-care practices in Nigerian retirees: a quasiexperimental study. Arch Public Health 2019;77:23.

33 Liu X, Hermalin Al, Chuang YL. The effect of education on mortality among older Taiwanese and its pathways. J Gerontol B Psychol Sci Soc Sci 1998;53:S71-82.

34 Chiang C-E, Wang T-D, Ueng K-C, et al. 2015 guidelines of the Taiwan Society of Cardiology and the Taiwan Hypertension Society for the management of hypertension. J Chin Med Assoc 2015;78:1-47. 
35 Oladipo I, Ayoade A. The effect of the first office blood pressure reading on hypertension-related clinical decisions. Cardiovasc J Afr 2012;23:456-62.
36 Salazar MR, Espeche WG, Aizpurúa M, et al. Should the first blood pressure reading be discarded? J Hum Hypertens 2015;29:373-8. 